\title{
An accelerated life evaluation method under multi - stress based on Eyring model
}

\author{
Shen Zhong-hong ${ }^{1, a}$, Liu Ju Lu $^{2, b}$ \\ ${ }^{1}$ The fifth research institute of MIIT. P. R. China, Guang Zhou, 510610 \\ ${ }^{2}$ The fifth research institute of MIIT. P. R. China, Guang Zhou, 510610 \\ aszh@ceprei.biz, ${ }^{b}$ lju@ceprei.biz
}

\begin{abstract}
Keywords: Accelerated life test; Accelerated model; Multi - stress; Eyring model
Abstract. How to obtain parameters of accelerated model is a key point in accelerated life testing under multi-stress. This paper proposed an accelerated life assessment method under multi - stress based on Eyring model by using vibration and temperature stress. The interactions between vibration and temperature in accelerated life test were researched in depth. And a conclusion was obtained that the temperature and vibration stress could be considered both independent in engineering applications approximately for their small interactions to each other. Some tests were implemented to realize and verify this method, which had a good performance.
\end{abstract}

\section{Introduction}

Reliability life testing and accelerated life testing have been widely used at home and abroad, as life characteristics, failure rule, MTBF of products can be obtained via it. Accelerated life testing is a useful experimental method to obtain products life features at nominal stress level, which based on the use of statistical models related to physical laws for conversion data information under accelerated environment beyond the normal stress via reasonable basis for carrying out projects and statistical assumptions [1].

Accelerated life testing can be divided into single- stress and multi-stress accelerated life testing according to the amount of stress applied on the accelerated life testing. Single-stress acceleration model mainly includes Arrhenius model and inverse power law model. Arrhenius model is widely used in the case of product life is the function of temperature, which accelerated stress is temperature only. At this time, the failure is caused by chemical reaction or metal diffusion. Inverse power law model is widely used in metal fatigue tests under mechanical stress or thermal stress cycles, in voltage durability test, bearings and incandescent life tests. Its characteristic is accelerated stress may be temperature or a non-temperature factor.Literature [2] discusses the derivation of relationship between linear life dependence Arrhenius model and temperature in detail, the meaning of the activation energy and parameters B, which laid the foundation for wide application of Arrhenius model. Literature [3,4] studied the implementation of Arrhenius model in different product life assessment. Literature[5] discussed the life distributions of progressive stress accelerated life tests for Weibuil distributions under TFR model and gave the Bayesian estimates of the distribution parameters and coefficients in the inverse power law accelerated equation. Literature [5] discussed the life distributions of progressive stress accelerated life tests for Weibuil distributions under TFR model and gave the Bayesian estimates of the distribution parameters and coefficients in the inverse power law accelerated equation.

In many cases, the effect of single stress acceleration is not obvious, multi-stress should be used to generate more acceleration effect. For multi-stress accelerated life test, its accelerated life model has multiple unknown parameters, we need to study new methods of data analysis. Paper [6] studied the statistical analysis of stress accelerated life test data of aerospace connectors under temperature and vibration testing stresses, but did not research how to obtain the relevant parameters of accelerated model of temperature and vibration stress. Paper [7] studied the accelerated life model under temperature and vibration stress comprehensive action and verified the Eyring model under temperature and vibration stress combined, but did not research the parameters acquisition method in 
acceleration model under temperature and vibration. Based on this, this paper use Eyring model as an example to study how to obtain model parameters of multi-stress accelerated life testing deeply.

\section{An accelerated life evaluation method in multi - stress based on Eyring model}

\section{Fundamental.}

Eyring model describes the relations between product life and stress under temperature stress $\mathrm{T}$ and non- temperature stress $S[8,9]$ :

$$
L=A S^{-\alpha} \exp ^{(B / T)}
$$

The linear model is :

$$
\ln L=A+B(1 / T)-\alpha \ln S
$$

\section{Acceleration factor is}

$$
A_{L}=\left(S / S_{N}\right)^{\alpha} \exp ^{[B \Delta(1 / T)]}
$$

Eyring model can be used on acceleration test of fatigue damage of mechanical parts, voltage resistance damage of insulation material, and power corrosion of the semiconductor device in temperature environment.

The accelerated life model has many parameters due to multiply stress working, typically three or more, $\left\{\mathrm{A}^{\prime}, \alpha, \mathrm{B}\right\}$. But the unknown parameters of acceleration factor model is two: 1 in temperature and 1 in non-temperature. Wherein the temperature effect parameter $\mathrm{B}=-\mathrm{E} / \mathrm{k}, \mathrm{E}$ is activation energy products, and $\mathrm{k}$ is Boltzmann's constant.

Accelerated life testing of electronic products usually used vibration and temperature stress combined. The destruction mechanisms of vibration for products are mechanical deformation, connector loose, structural damage, etc., wherein the specific failure modes of structural damage includes open circuit, short circuit, wire break, void welding, open welding, crystal defects, surface wear. The product failure modes caused by temperature include parameter drift, physical changes, electro migration, seal failure, accelerated oxidation (metallization) and so on.

The interaction of temperature and vibration is shown in Fig 1. The influence generated by temperature for the failure mechanism of vibration performances oxidation corrosion of the metal surface. Adhesion friction occurs when micro vibration generated between the mechanical parts in contact with each other, which resulting in surface wear of mechanical parts, and wear debris and mechanical components surfaces exposed are oxidated rapidly in high temperature to form an oxide corrosion, which making product failure caused by contact resistance increasing. However, this acceleration is one of the failure mechanism of temperature for the product itself [10]. It can regarded as the effects of temperature for the vibration acceleration is small; and vibration have no effect for temperature acceleration. Therefore, temperature and vibration can be considered independent in engineering applications approximately, and the acceleration effects can be considered separately. 


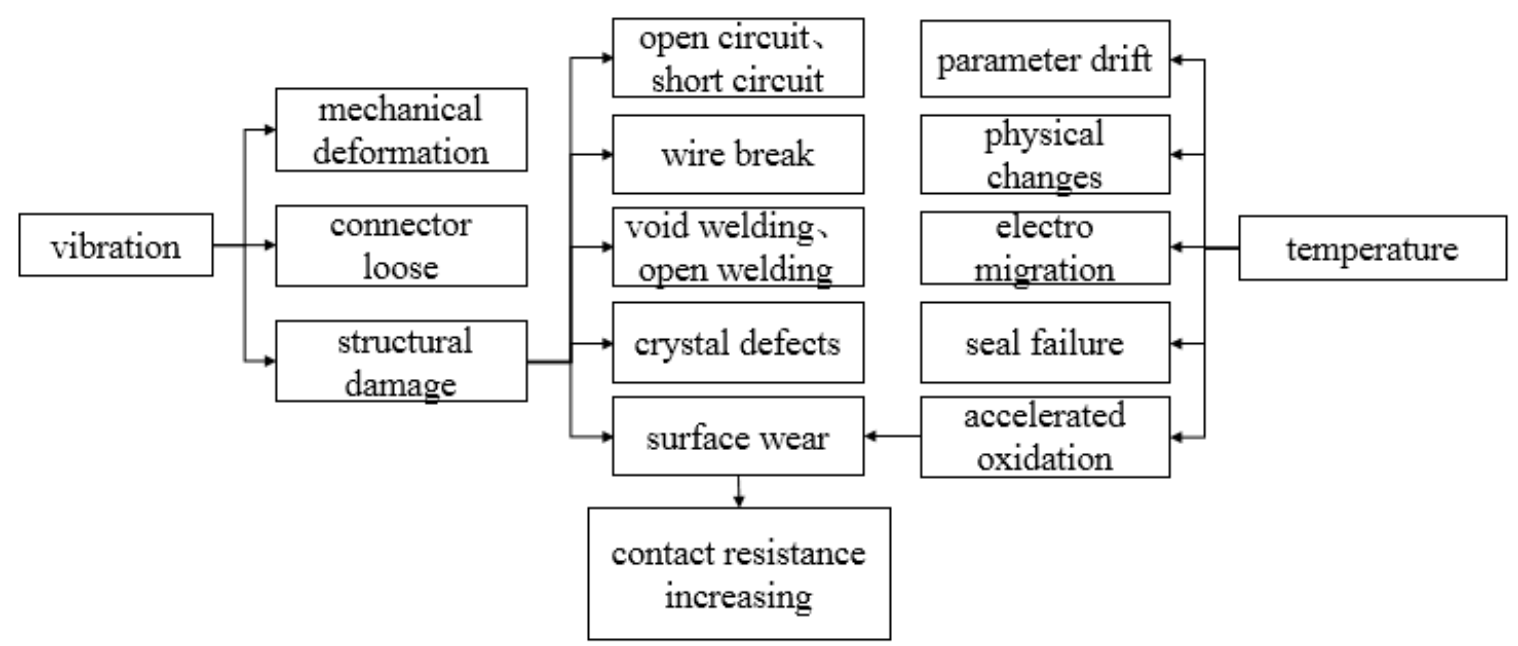

Fig. 1 The interaction between vibration and temperature stress in accelerated testing

Algorithm principle. When the acceleration effects of temperature and vibration can be considered separately, the relationship between temperature and product life, vibration and product life can be separated in Eyring model. Assume L1 is the product life when the vibration stress applied, L2 is the product life when the temperature stress applied, then:

$$
\begin{aligned}
& L_{1}=A_{1} S^{-\alpha} \\
& L_{2}=A_{2} \exp (B / T)
\end{aligned}
$$

Taking the natural logarithm on both sides of the formula (4), then have:

$$
\ln L_{1}=\ln A_{1}+(-\alpha) \ln S
$$

The parameters of formula (6) can be obtained in logarithmic vibration stress axes and logarithmic life axes based on the failure data at different levels of vibration stress, and the accelerated life model of the product can be obtained under vibration stress, which is shown in Fig. 2(a).

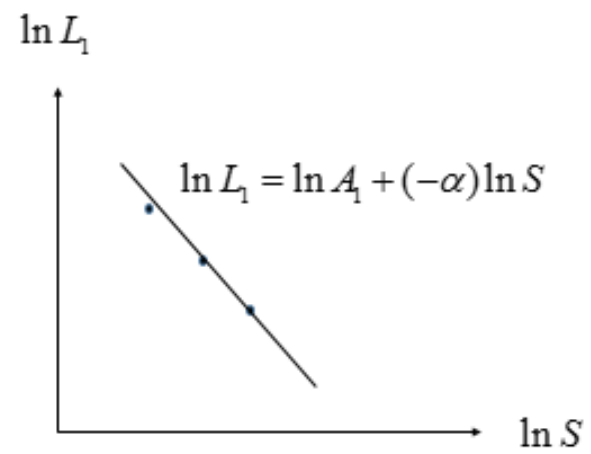

(a)

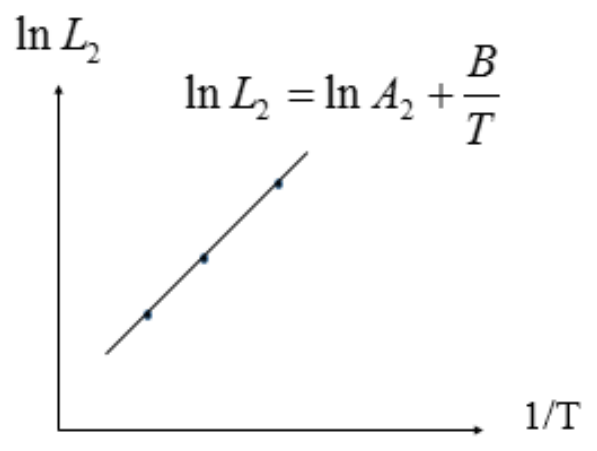

(b)

Fig. 2 the methods to obtain the parameters of accelerated life model

Taking the natural logarithm on both sides of the formula (5), which indicates accelerated testing under temperature stress, we have:

$$
\ln L_{2}=\ln A_{2}+\frac{B}{T}
$$


The parameters of formula (7) can be obtained in reciprocal temperature axis and logarithmic life axes based on the failure data at different levels of temperature stress, and the accelerated life model of the product can be obtained under temperature stress, which shown in Fig. 2(b).

\section{Tests}

Tests were implemented to verify the accelerated life evaluation method based on Eyring model by using vibration and temperature stress in this paper. According to preliminary HALT test to determine the temperature and vibration stress levels of accelerated life test. Three samples were used at each stress level for testing, and the experimental data obtained are as follows:

Table 1 the experimental data

\begin{tabular}{|l|c|c|c|c|}
\hline \multicolumn{2}{|c|}{ Vibration $\left(\mathrm{G}_{\mathrm{rms}}\right)$} & $\mathrm{S} 1$ & $\mathrm{~S} 2$ & $\mathrm{~S} 3$ \\
\hline \multicolumn{2}{|c|}{ Failure time $(\mathrm{h})$} & & & 48 \\
\hline \multicolumn{2}{|c|}{ Temperature $\left({ }^{\circ} \mathrm{C}\right)$} & 35 & 41 & 12.1 \\
\hline \multirow{2}{*}{ T1 } & \multirow{2}{*}{85} & $/$ & $/$ & 12.6 \\
\cline { 3 - 5 } & & $/$ & $/$ & 12.7 \\
\cline { 3 - 5 } & \multirow{2}{*}{ T2 } & $/$ & $/$ & 8.3 \\
\cline { 3 - 5 } & \multirow{3}{*}{102} & $/$ & $/$ & 8.5 \\
\cline { 3 - 5 } & & 14.2 & 8.5 & 9.2 \\
\hline \multirow{2}{*}{ T3 } & 14.8 & 8.8 & 6.1 \\
\cline { 3 - 5 } & & 15.3 & 9.3 & 6.3 \\
\hline
\end{tabular}

Weibull distribution model can be used for machinery and electronic products life evaluation for it can represent the failure laws of devices and integrated circuits well, can fully reflect the impact of material defects and stress concentration source for material fatigue life, and have increasing failure rate. Assume the life distribution used in tests is a two-parameter Weibull distribution, then the distribution function is:

$$
F(t)=P(T<t)= \begin{cases}1-e^{-\left(\frac{t}{\eta}\right)^{m}} & t \geq 0 \\ 0 & t<0\end{cases}
$$

Wherein, $\mathrm{m}(>0)$ is shape parameter, $\eta(>0)$ is features life.

According to the test data in Table 1, to maintain vibration stress constant and change the temperature, resulting in 9 groups data to study the temperature acceleration model by using the Arrhenius Celsius temperature model.

$$
A F_{1}=\exp ^{\frac{E}{k}\left(\frac{1}{T_{1}}-\frac{1}{T_{s}}\right)}
$$

The life data distribution model based on experimental was shown in Fig. 3.(a), the life temperature stress model was shown in Fig. 3(b). 


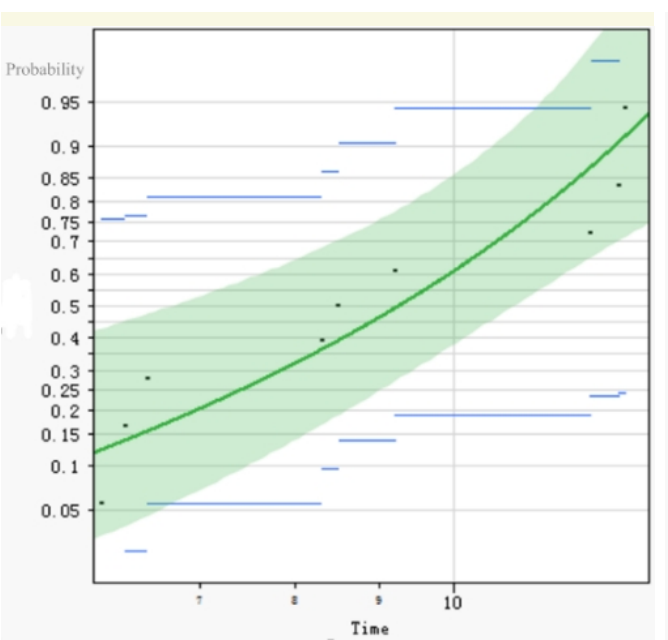

(a)

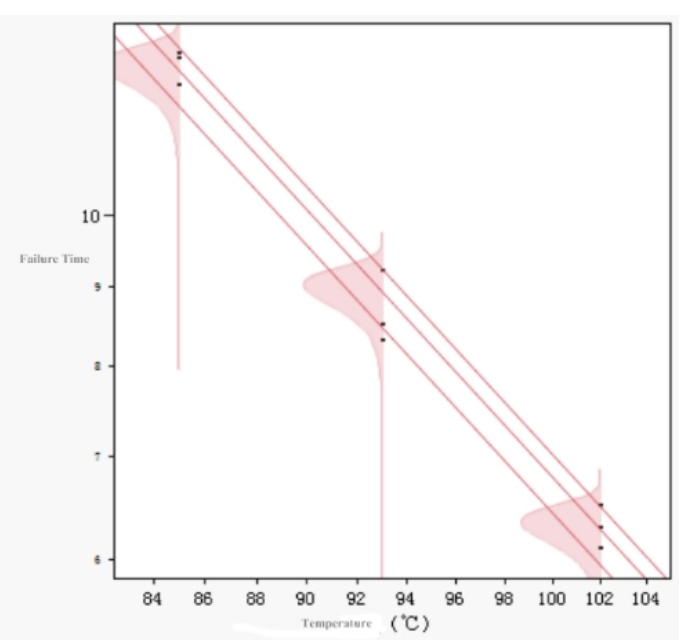

(b)

Fig. 3 the temperature experimental data research

According to test data, the activation energy parameters of Arrhenius model $\mathrm{E}$ is $0.4617 \mathrm{eV}$, and the accelerated coefficients B is 5358 in this accelerated model.

The same, to maintain temperature stress constant and change the vibration stress, resulting in 9 groups data to study the vibration acceleration model. The life data distribution model based on experimental was shown in Fig. 4(a), the life - vibration stress model was shown in Fig. 4(b).

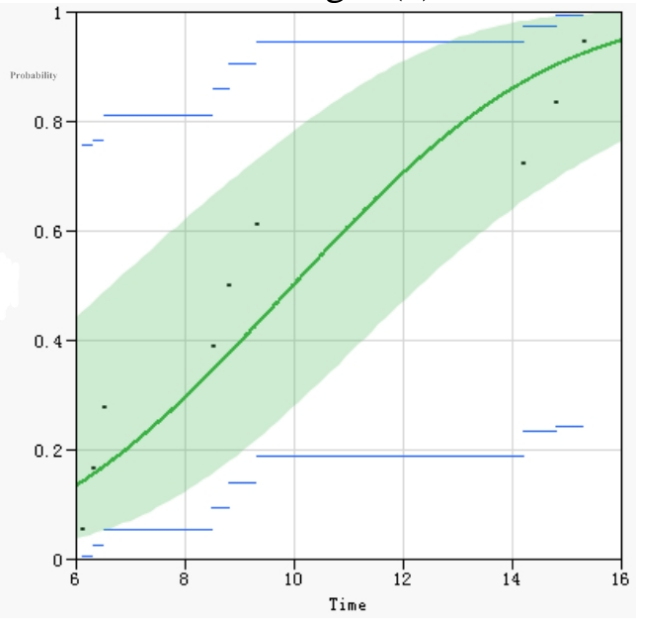

(a)

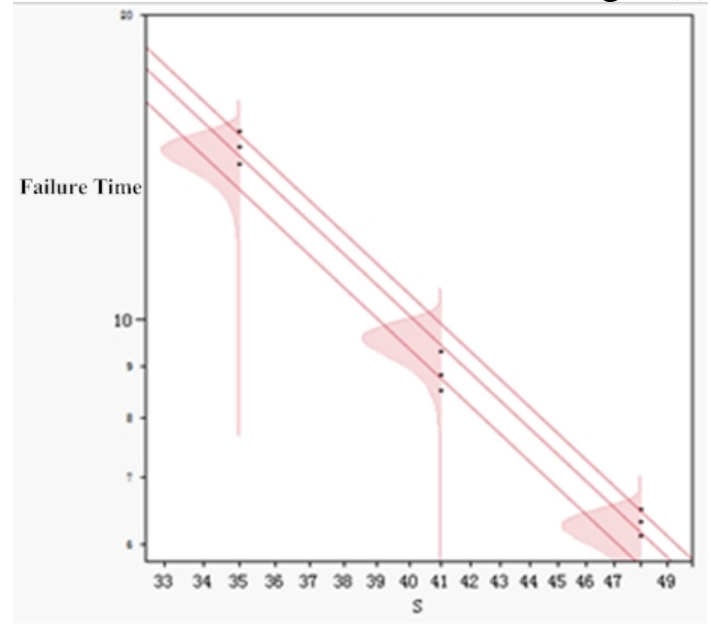

(b)

Fig. 4 the vibration experimental data research

According to test data, the parameter $\alpha$ of vibration acceleration model (natural logarithm model) is 2.704807 .

\section{Summary}

In this paper, accelerated life assessment methods under multi - stress based on Eyring model was researched by using vibration and temperature stress. The interactions between vibration and temperature in accelerated life test were analyzed in depth, and the accelerating effects of these two stress could be regarded as independent in engineering applications approximately. Some tests were implemented to verify this method, which showed it had good effect and could be applied in the accelerated life testing of electronic products.

\section{Acknowledgements}

This work was financially supported by the Applied Science Technology Research Foundation of Guangdong Province (2015B010133001), Major Science and Technology Research Foundation of 
Guangdong Province (2016B010113004), Guangdong Provincial Science and Technology Project (2015B090906022).

\section{References}

[1] W. Yurkowsky, R. E. Schafter, J. M. Finkelstein. Accelerated testing technology [J]. Accelerated Testing Technology, 1967:1-2.

[2] L. Zhen, J. T. Min, C. Y. Sheng, et al. Study on Arrhenius relationship [J]. Electronic product reliability and environmental testing, 2005, 6: 12-14.

[3] M. Edirisinghe, P. Rathnayake. Arrhenius Accelerated Life Test for Luminary Life of High Bright Light Emitting Diodes[C]. International Letters of Chemistry, Physics and Astronomy,2015,49:48-59.

[4] P. Espinet-González, C. Algora, N. Núñez, et al. Temperature accelerated life test on commercial concentrator III-V triple-junction solar cells and reliability analysis as a function of the operating temperature[J]. Progress in Photovoltaics Research \& Applications, 2015, 23(5):559-569.

[5] T. Y. Cai, L. F. Fang. Bayesian Analysis of Weibull Progressive Stress Accelerated Life Tests under TFR and Inverse Power Law Models [J]. Journal of Shanghai Normal University (Natural Science), 2014, 1: 26-31.

[6] C. W. Hua, L. H. Shi, L. W. Zhi, et al. Accelerated life test and statistical analysis of aerospace electrical connectors under multiple environmental stresses [J]. Journal of Zhejiang University (Engineering Science), 2006, 40 (2):348-351.

[7] Q. Ping, C. W. Hua, M. Z. kui, et al. Research of multiple stresses accelerated life model Verification method [J]. Journal of Mechanical Engineering, 2010, 46 (24):156-161.

[8] Laidler, K. James. The theory of rate processes [M]. McGraw-Hill Book Company, 1941.

[9] M. S. Song. The accelerated model of accelerated testing [J]. Quality and Reliabity, 2003(2):15-17.

[10] Z. X. Ping, X. K. Yi, J. W. Yuan, et al. The effect of metal oxide film at different temperature on flyash erosion [J]. J. East University of Technology, 1996(1):73-79. 